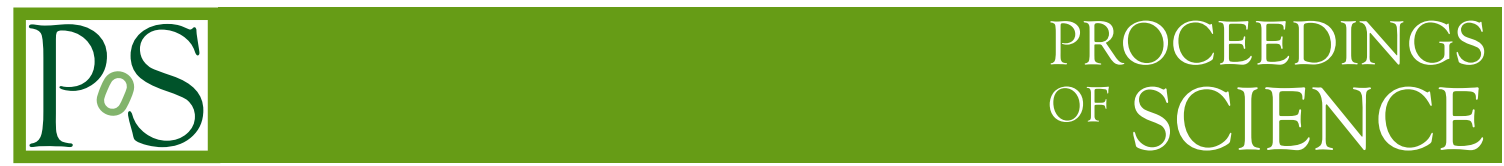

\title{
On ultra-high energy cosmic rays
}

\section{Martin Lemoine*}

Institut d'Astrophysique de Paris

CNRS - Sorbonne Université

98 bis boulevard Arago, F-75014 Paris, France

E-mail: lemoinediap.fr

This paper summarizes some results on our understanding of the origin of ultra-high energy cosmic rays.

Neutrino Oscillation Workshop (NOW2018)

9 - 16 September, 2018

Rosa Marina (Ostuni, Brindisi, Italy)

${ }^{*}$ Speaker. 


\section{Constraints on the sources}

How ultra-high energy cosmic rays (UHECR) can be accelerated to energies $\gtrsim 10^{20} \mathrm{eV}$ and in what astrophysical source, remains a long-standing enigma of high-energy astroparticle physics. The development of gigantic experiments in recent decades has permitted key advances, in particular the detection of the high-energy cut-off of the Greisen-Zatsepin-Kuzmin (GZK) effect, at an energy $E_{\mathrm{GZK}} \simeq 5 \times 10^{19} \mathrm{eV}$, where the Universe becomes opaque to ultra-high energy protons or nuclei due to photohadronic interactions off diffuse radiative backgrounds ${ }^{1}$.

\subsection{Particle acceleration}

To appreciate the challenge of accelerating particles up to GZK energies, consider the following relativistic generalization of the Hillas bound [1], e.g. [2] and references therein. Writing the timescale of particle acceleration in the source comoving frame as $t_{\mathrm{acc}} \equiv \mathscr{A} t_{\mathrm{g}}$, and bounding it by above with the dynamical timescale ${ }^{2}, t_{\mathrm{dyn}}=R /(\Gamma \beta c)$, one derives a lower bound on the magnetic luminosity of the source to produce particles of charge $Z$ and energy $E_{20} 10^{20} \mathrm{eV}$ :

$$
L_{B} \gtrsim 10^{45} \mathscr{A}^{2}\left(E_{20} / Z\right)^{2} \mathrm{erg} / \mathrm{s} .
$$

As discussed in the above reference, this bound is robust and only a few types of rare sources exhibit luminosities in excess of $10^{45} \mathrm{erg} / \mathrm{s}$ : high luminosity explosive sources such as gamma-ray bursts, highly powerful radio-galaxies and possibly pulsars or magnetars born with a msec rotation period. Of course, if $Z \gg 1$, the lower bound on the luminosity is significantly reduced, because what truly matters is the rigidity $E /(e Z)$.

The theory of particle acceleration shows that $\mathscr{A}>1$ and actually $\mathscr{A} \gg 1$ is typical. For instance, the generic Fermi-type scenarios predict $\mathscr{A} \sim g(E) / \beta_{\mathrm{F}}^{2}$, where $\beta_{\mathrm{F}}$ represents the typical velocity (in units of $c$ ) of moving scattering centers (or a shock velocity for Fermi-I), and $g(E)=$ $t_{\text {scatt }} / t_{\mathrm{g}}>1$ denotes the ratio of the scattering timescale in the turbulence to the gyro-time. The so-called Bohm regime, $g(E) \sim 1$, which is often used, represents an idealized situation in which particles scatter by an angle of the order of unity at each gyro-period, see [3].

Note that radio-galaxies with luminosity above $10^{45} \mathrm{erg} / \mathrm{s}$ come with a density $n_{>45} \sim 10^{-7} / \mathrm{Mpc}^{3}$, while $n_{>47} \sim 10^{-11} / \mathrm{Mpc}^{3}$ for luminosities in excess of $10^{47} \mathrm{erg} / \mathrm{s}$. Such objects are simply too rare to exist in the GZK-sphere and thus to produce particles at GZK energies. Hence, if radio-galaxies are to accelerate ultra-high energy cosmic rays, these cosmic rays must be intermediate or heavy mass nuclei, i.e. $Z>1$, in order to reduce the energy requirements. A potential exception is the scenario of accelerating particles in the central black hole magnetosphere, which escapes the above bound, see [4].

\subsection{Source characteristics}

Another constraint that can be derived on the source of UHECR is a lower bound on their apparent density: $n_{\mathrm{s}} \gtrsim 10^{-6}-10^{-4} \mathrm{Mpc}^{-3}$. This density is termed apparent because of the possible

\footnotetext{
${ }^{1}$ Strictly speaking, one cannot exclude that this cut-off rather attests of an intrinsic limitation of sources in accelerating particles to higher energies.

${ }^{2} t_{\mathrm{g}}$ : gyrotime; $R$ : distance to center of expansion at bulk velocity $\beta c$ with bulk Lorentz factor $\Gamma$.
} 
effect of intervening magnetic fields on the trajectories of UHECR, e.g. [5]. This bound on the density derives from the near isotropic and non-repeating pattern of arrival directions, more specifically from the lack of multiplets of events which would be expected for lower source densities [6].

Another constraint bounds by below the energy output per source in UHECR. The comparatively short lifetime of GZK particles implies that their reservoir in the intergalactic space must be replenished at a high rate, of the order of $\dot{\varepsilon} \sim 10^{44} \mathrm{erg} / \mathrm{Mpc}^{3} / \mathrm{yr}$ [7]. In the case of gamma-ray bursts, with $\dot{n}_{\mathrm{s}} \sim 10^{-9} / \mathrm{Mpc}^{3} / \mathrm{yr}$, this necessitates $10^{53} \mathrm{erg}$ in UHECR [8]. For radio-galaxies of density $n_{\mathrm{S}}=10^{-7} n_{-7} \mathrm{Mpc}^{-3}$, this requires $L_{\mathrm{UHECR}} \sim 3 \times 10^{43} n_{-7}^{-1} \mathrm{erg} / \mathrm{s}$, i.e. a conversion efficiency of already 3\%! Considering the whole population of radio-galaxies, as e.g. in [9] and assuming that ultra-high energy cosmic rays are heavy nuclei so as to weaken the luminosity constraint, one also derives a conversion factor of a few percent, when accounting for the concomittant injection of protons at energies $\gtrsim 10^{18} \mathrm{eV}$... The source must therefore channel a sizable fraction of its energy output into the non-thermal population at the highest energies.

\subsection{Chemical composition}

The chemical composition remains poorly known at the highest energies, but recent results by the Pierre Auger Observatory indicate a shift from a light, proton-like composition at $10^{18} \mathrm{eV}$ energies to an intermediate composition at sub-GZK energies. This uncertainty is rather embarrassing, because the chemical composition controls most of the phenomenology of this field of research:

- at a given energy $E$, say GZK, the rigidity of a heavy nucleus of charge $Z$ is $Z$ times smaller than that of a proton; it is thus deflected by angle $Z$ times larger, hence the prospects for detecting anisotropies appear dire.

- as discussed above, acceleration scales with rigidity, hence it is somewhat less of a challenge to find a source of $10^{20} \mathrm{eV}$ heavy nuclei than to find a source of $10^{20} \mathrm{eV}$ protons, meaning that the pool of candidates for ultra-high energy nuclei is quite substantially larger than that for protons.

- multi-messenger astronomy is also more pessimistic for heavy nuclei, which produce significantly less secondary messengers (e.g. neutrinos, photons) than light nuclei of a same energy, e.g. [10].

\subsection{Anisotropies}

Interestingly, recent experimental results reveal the existence of anisotropies on various scales, even at GZK energies, e.g. the Telescope Array hotspot [11] or the recent Pierre Auger correlation with high-energy source catalogs [12]. As discussed in [2], such anisotropies offer a robust onthe-sky test of the chemical composition of UHECR, as follows.

If the anisotropies at $E_{\mathrm{GZK}}$ energies are produced by nuclei of charge $Z>1$, one should observe a stronger anisotropy signal at energies $E_{\mathrm{GZK}} / Z$, which is associated with the protons of a same rigidity (but smaller energy) emitted by the source [2]. More specifically, defining the signalto-noise ratio $\Sigma_{Z}(E)$ of the anisotropy, one can show that the signal-to-noise ratio for protons at $E_{\mathrm{GZK}} / Z$ is: $\Sigma_{p}(E / Z) \gtrsim Z^{-0.85} \frac{N_{p}}{N_{Z}} \Sigma_{Z}(E)$. Since $N_{p} / N_{Z}$, the ratio of the number of protons to nuclei emitted by the source, is expected to be $\gg 1, \Sigma_{p}(E / Z) \gg \Sigma_{Z}(E)$. Hence the above anisotropy searches should be complemented by searches at lower energies, in the same direction.

The Pierre Auger collaboration has performed this test and found a null result at lower energies [13]. According to [14], this result indicates that: either the anisotropy pattern at GZK is not 
produced by heavy nuclei, but by light nuclei. It could mean that at these energies, the composition becomes dominated by light nuclei, or that it remains dominated by intermediate or heavy mass nuclei, and a subset of protons produce these anisotropy patterns. Or, if the anisotropy pattern is associated to heavy nuclei, the number of injected protons must be small, meaning that the metallicity of the source must be $\gtrsim 100$ times the solar metallicity for CNO type nuclei, and $\gtrsim 1000$ solar for heavier nuclei.

Acknowledgements: This work has been financially supported by the ANR-14-CE33-0019 $\mathrm{MACH}$ project.

\section{References}

[1] A. M. Hillas, The Origin of Ultra-High-Energy Cosmic Rays, Ann. Rev. Astron. Astrophys. 22, 425 (1984).

[2] M. Lemoine, E. Waxman, Anisotropy vs chemical composition at ultra-high energies, J. Cosm. Astropart. Phys. 11009 (2009).

[3] F. Casse, M. Lemoine, G. Pelletier, Transport of cosmic rays in chaotic magnetic fields, Phys. Rev. D65, 023002 (2002).

[4] A. Levinson, Particle Acceleration and Curvature TeV Emission by Rotating, Supermassive Black Holes, Phys. Rev. Lett. 85, 912 (2000).

[5] K. Kotera, M. Lemoine, Optical depth of the Universe to ultrahigh energy cosmic ray scattering in the magnetized large scale structure, Phys. Rev. D77, 123003 (2008).

[6] Pierre Auger Collaboration, Search for patterns by combining cosmic-ray energy and arrival directions at the Pierre Auger Observatory, Eur. Phys. J. C75, 269 (2015).

[7] B. Katz, R. Budnik, E. Waxman, The energy production rate \& the generation spectrum of UHECRs, J. Cosm. Astropart. Phys. 3020 (2009).

[8] E. Waxman, Cosmological Gamma-Ray Bursts and the Highest Energy Cosmic Rays, Phys. Rev. Lett. 75 386-389 (1995).

[9] V. Ptuskin, S. Rogovaya, V. Zirakashvili, On ultra-high energy cosmic rays: Origin in AGN jets and transport in expanding universe, Adv. Sp. Res. 51 315-321 (2013).

[10] M. Ahlers, F. Halzen, Minimal cosmogenic neutrinos, Phys. Rev. D86, 083010 (2012).

[11] R. U. Abbasi, et al. (Telescope Array Collaboration), Indications of Intermediate-scale Anisotropy of Cosmic Rays with Energy Greater Than 57 EeV in the Northern Sky Measured with the Surface Detector of the Telescope Array Experiment, Astrophys. J. 790 L21 (2014).

[12] Pierre Auger Collaboration, Indication of anisotropy in arrival directions of ultra-high-energy cosmic rays through comparison to the flux pattern of extragalactic gamma-ray sources, Astrophys. J. 835, L29 (2018).

[13] Pierre Auger Collaboration, Anisotropy and chemical composition of ultra-high energy cosmic rays using arrival directions measured by the Pierre Auger Observatory, J. Cosm. Astropart. Phys. 06, 022 (2011).

[14] R.-Y. Liu, A. M. Taylor, M. Lemoine, X.-Y. Wang, E. Waxman, Constraints on the Source of Ultra-high-energy Cosmic Rays Using Anisotropy versus Chemical Composition, Astrophys. J. 77688 (2013). 